\title{
Théologiques
}

Théologiques

\section{L'autre du corps dans la Bible}

\section{Olivette Genest}

Volume 5, numéro 2, octobre 1997

Le corps : du dualisme à l'altérité

URI : https://id.erudit.org/iderudit/024948ar

DOI : https://doi.org/10.7202/024948ar

Aller au sommaire du numéro

Éditeur(s)

Faculté de théologie de l'Université de Montréal

ISSN

1188-7109 (imprimé)

1492-1413 (numérique)

Découvrir la revue

Citer cet article

Genest, O. (1997). L'autre du corps dans la Bible. Théologiques, 5(2), 51-70.

https://doi.org/10.7202/024948ar

\section{Résumé de l'article}

La vision de la personne humaine mise en discours dans la Bible ne pose pas un composé de corps et d'âme mais un être que le vocabulaire pertinent décrit sous différents angles de perception. Ainsi le corps désigne-t-il le sujet humain entier, sous un aspect à déterminer par les contextes et non par les définitions d'une anthropologie étrangère au texte. Une relecture en ce sens de déclarations de Paul et de l'expérience corporelle de Jésus dans les récits évangéliques révèle l'inadéquation de certaines de nos réflexions théologiques sur « les choses de la chair ». Elle pose aussi la question du statut de l'anthropologie hébraïque dans les discours que nous construisons à partir de la Bible.
Tous droits réservés @ Faculté de théologie de l'Université de Montréal, 1997
Ce document est protégé par la loi sur le droit d'auteur. L'utilisation des services d'Érudit (y compris la reproduction) est assujettie à sa politique d'utilisation que vous pouvez consulter en ligne.

https://apropos.erudit.org/fr/usagers/politique-dutilisation/ 


\title{
L'autre du corps dans la Bible
}

\author{
Olivette GENEST \\ Faculté de théologie \\ Université de Montréal
}

\section{RÉSUMÉ}

La vision de la personne humaine mise en discours dans la Bible ne pose pas un composé de corps et d'âme mais un être que le vocabulaire pertinent décrit sous différents angles de perception. Ainsi le corps désigne-t-il le sujet humain entier, sous un aspect à déterminer par les contextes et non par les définitions d'une anthropologie étrangère au texte. Une relecture en ce sens de déclarations de Paul et de l'expérience corporelle de Jésus dans les récits évangéliques révèle l'inadéquation de certaines de nos réflexions théologiques sur " les choses de la chair". Elle pose aussi la question du statur de l'anthropologie hébraïque dans les discours que nous construisons à partir de la Bible.

The vision of the human person in Biblical discourse does not present a composite of body and soul, but a being that the pertinent vocabulary describes under different angles of perception. Thus the body designates the whole human subject, under the aspects to be determined by the contexts and not by the definitions of an anthropology foreign to the text. A re-reading in this sense of the declarations of Paul and of the corporeal experience of Jesus in the gospel narratives reveals the inadequacy of some of our theological reflections on " matters of the flesh. "It also poses the question of the status of Hebraic anthropology in the discourses that we construct from the Bible.

Dans notre univers conceptuel, nous entendons corps/âme, corps/esprit, corps/raison, la catégorie matériel/spirituel et les qualifications qui opposent les deux pôles de ces dichotomies où percent hiérarchie, axiologisation, dévalorisation et sur-valorisation. Les horizons du pays biblique ne rendent pas les mêmes échos. L'autre par excellence du corps, l'âme, est absente de la langue hébraïque, support linguistique de l'Ancien Testament, mais présente dans la langue grecque, instrument rédactionnel du Nouveau Testament. Le mot corps lui-même n'existe pas en hébreu mais est courant en grec. Le grec de la Septante qui devait 
calquer scrupuleusement l'hébreu vétérotestamentaire se laisse parfois emporter par le génie hellénistique. La phrase grecque du Nouveau Testament a souvent été pensée en hébreu ou en araméen. L'ensemble de la Bible chrétienne nous est parvenu à travers des lunettes aristotélicothomistes. Enfin, le magistère catholique adhère toujours à l'anthropologie thomiste d'origine grecque, ce qui n'est pas le cas dans l'éventail des dénominations protestantes.

À travers ces méandres des langues, de l'écriture et de la lecture, il n'en demeure pas moins que le corps, et ce à quoi il renvoie en hébreu, n'a pas d'autre dans la Bible, d'autre interne à la personne humaine. Il n'est pas une composante de l'unité individuelle, même si on en faisait une partie égale en dignité à l'âme, à l'esprit, à la raison, un instrument aussi noble que la partie dirigeante du tout. En stricte logique biblique, je ne peux parler d'un moi réfléchissant sur sa condition corporelle. Je n'ai pas un corps, je suis un corps. Ce je corporel peut réfléchir sur sa condition spécifique. Et la condition corporelle, l'expérience corporelle, c'est, en termes bibliques, la vie humaine elle-même.

La naissance "corporelle " ne consiste pas en l'arrivée d'une âme dans un corps matériel, sauf dans certains passages des écrits sapientiaux qui reflètent une contamination hellénistique que s'empresseront de corriger le judaïsme rabbinique et le Nouveau Testament. Le cours de l'existence ne s'exprime pas comme la durée d'un corps qui change et s'use, portant une âme stable, invulnérable, dépositaire de l'identité et du perfectionnement de l'individu humain. La mort corporelle, c'est la mort de toute la personne; la résurrection de même. À la grecque, le credo catholique parle plutôt d'un corps qui meurt et qui ressuscite, face à une âme, son âme, qui subsiste, elle, immortelle. On connaît toutes les variantes et les ambiguïtés que soulève cette conception.

La justification et les nuances de ces affirmations globales nous conduiront ici à une incursion chez Paul et dans les évangiles. Nous y trouverons deux séries de conclusions d'inégales longueurs: 1) le corps: le sujet humain; 2) l'autre du corps : l'intersubjectivité et l'être chrétien, ou l'existence "en un Corps». Nous leur poserons la question de l'utilité, ou de l'embarras, de ces précisions exégétiques pour nous qui sommes issus de la pensée grecque sur le corps.

\section{Le corps : le sujet humain}

\subsection{Les lettres de Paul}

Le corpus paulinien constitue le meilleur champ d'observation sur la conception du corps au début du christianisme. Lettres authentiques et 
paraphrases attribuées à l'apôtre, retenues par la tradition sous le sceau de son autorité, portent la marque du personnage Paul. À la différence des évangiles, cette signature unique assure la cohérence de la vision de l'humanité véhiculée sur et entre leurs lignes. Cohérence ne signifie pas pour autant simplicité. Personnage complexe, Paul participe de deux cultures, la juive et l'hellénique, de par son lieu d'origine et son éducation. Trois anthropologies s'entremêlent dans ses écrits: l'hébraïque, la grecque et celle qu'il s'est donnée pour rendre compte de la transformation de son existence par le Christ.

Si l'on s'en tenait à la salutation finale de sa première lettre : "Que le Dieu de la paix lui-même vous sanctifie totalement et que votre être entier, l'esprit, l'âme et le corps, soit gardé sans reproche à l'avénement de notre seigneur Jésus Christ " $(1 \mathrm{Th} 5,23)^{1}$, Paul posséderait une conception tripartite de la personne humaine. La suite de sa correspondance va développer un panorama tout autre, dont devra tenir compte la lecture de 1 Th 5,23.

Bultmann a rendu familiers les principaux termes de l'anthropologie paulinienne ${ }^{2}$ : psuchè, nous, sôma, sarx et pneuma. Les contextes de leurs emplois nous apprennent rapidement que Paul déroge cependant au lexique de la langue grecque. Des signifiants grecs recouvrent des signifiés hébraïques et une charge sémantique relevant de l'anthropologie hébraïque. Psuchè ne signifie pas l'âme à la grecque mais la néfesh à l'hébraïque, c'est-à-dire le vivant, humain ou animal. Nous, intelligence et raison renvoie à lèv ou cœur, siège de la pensée chez les Hébreux, sarx, ou chair, à bâsâr, c'est-à-dire à la personne humaine dans sa finitude, en tant qu'être fragile, soumis à l'usure et à la mort, susceptible d'erreur et d'errance, à la fidélité difficile, à la durée précaire. Avec pneuma, souffle et esprit, nous touchons à l'originalité de Paul : son pneuma chrétien ne correspond pas entièrement à la rouah ou souffle divin dans l'Ancien Testament. Enfin, sôma n'a pas d'équivalent dans l'anthropologie vétérotestamentaire, qui ne possède pas la distinction corps/âme, matériel/spirituel.

À ces termes, il faudrait en ajouter d'autres, tels ceux de suneidèsis (conscience) et phronèsis (à la traduction si subtile). La difficulté à clore la liste nous avertit déjà que la simple énumération ne suffit pas. Pour cerner

1 Traduction de la Bible de Jérusalem. Les notes explicatives dans les différentes bibles reflètent éloquemment les hésitations des traducteurs.

2 R. BULTMANN, Theologie des Neuen Testaments. Tübingen, J.C.B. Mohr (Paul Siebeck) 1948 et 1953. Traduction anglaise: Theology of the New Testament, I-II, London, SCM, 1965. 
l'acception paulinienne de ces termes, faut-il encore tenir compte des adjectifs qu'il en tire (qu'est-ce que Paul déclare corporel, charnel?), de ce à quoi il les oppose, des qualifications qu'il leur octroie, que nous dirions physiques et morales. Il faut surtout les lire, en état de système, dans leur mise en discours.

Un passage de la lère aux Corinthiens servira de brève illustration et de relance de la question sur l'acception du corps chez Paul. L'exercice de lecture consistera à s'imposer le dépaysement de la conclusion tirée d'études sur le corpus paulinien entier : les termes anthropologiques rencontrés ne sont pas des éléments de la personne humaine, mais des aspects et des états de sa réalité globale, selon le point de vue adopté par l'observateur. La transcription ci-dessous restitue à la traduction française les mots grecs pertinents.

1 Co $15,35-50$ :

35 - Mais, dira-t-on, comment les morts ressuscitent-ils? avec quel sôma reviennent-ils? 36 - Insensé! Toi, ce que tu sèmes ne fait-vie qu'à condition de mourir. 37 - Et ce que tu sèmes n'est pas le sôma qui doit naître mais un grain nu, de blé ou d'autre chose. 38 - Puis Dieu lui donne sôma, comme il le veut et à chaque semence son propre sôma. 39 - Aucune sarx n'est identique à une autre sarx, mais autre celle des hommes, autre la sarx des bêtes, autre la sarx des oiseaux, autre celle des poissons. 40. Il y a des sômata célestes et des sômata terrestres, mais autre est l'éclat des célestes et autre celui des terrestres. 41 . Autre l'éclat du soleil et autre l'éclat de la lune et autre l'éclat des étoiles; une étoile même diffère en éclat d'une autre étoile. 42 - Il en est ainsi pour la résurrection des morts : le sôma semé dans la corruptibilité ressuscite dans l'incorruptibilité; 43 - semé dans l'absence d'honneur, il ressuscite dans la gloire; semé dans la faiblesse, il ressuscite dans la force; 44 - semé sôma psuchikon, il ressuscite sôma pneumatikon; s'il y a un sôma psuchikon, il y a aussi un sôma pneumatikon. 45 - Ainsi est-il écrit : "Le premier homme Adam fut (advint vers) une psuchè vivante, le dernier Adam un pneuma créateur-de-vie (litt. : faisant-la-vie). 46. Mais ce n'est pas le pneumatikon qui fut le premier, mais le psuchikon, puis le pneumatikon; 47 - Le premier homme est issu de la terre, le deuxième homme du ciel. 48 - Tel le terrestre, tels aussi les terrestres, et tel le céleste, tels aussi les célestes. 49 . Et de même que nous avons été à l'image de l'homme terrestre, nous serons aussi à l'image de l'homme céleste. 50. J'affirme ceci, frères: la sarx et le sang ne peuvent hériter du royaume de Dieu, ni la corruptibilité hériter de l'incorruptibilité. 
Une question à la grecque, sur l'horizon de la distinction corps/âme, comme nous la poserions à propos de la résurrection où le sort du corps est le plus énigmatique, attire à un "Toi » rhétorique une réponse qui est, elle, une trouvaille. Entre le grain semé et la plante qui en germe, le lien d'identité demeure dans la plus grande disparité de la forme du pôle de départ et du pôle d'arrivée. "Insensés » que nous sommes, comment n'avons-nous pas remarqué que, dans ce monde d'avant la mort, nous vivons, de toute façon, entourés d'une diversité de sôma et de sarx mirobolante, jusqu'à l'étoile qui diffère de l'étoile.

Des versets 35 à 41 , nous apprenons, au plan de l'emploi des termes, que sôma ou corps peut désigner les vivants et les non-vivants (classification qui inclut les végétaux dans la Bible), mais que sarx ou chair ne s'applique qu'aux vivants, ou néfesh: humains, animaux, oiseaux et poissons. Quand Paul revient à la résurrection des morts, au verset 42 , il ne garde que sôma. Le terme sarx disparaît, puisqu'il ne peut désigner que l'humain d'avant la résurrection, cette dernière effaçant définitivement l'aspect de finitude et de fragilité qu'elle exprimait. Paul ne parlera jamais, et pour cause, de "résurrection de la chair ".

Les versets 42 et 43 sont marqués par la persistance de l'emploi de sôma du côté de la résurrection elle-même. Un corps est semé et c'est un corps qui ressuscite. C'est-à-dire un être humain somatique meurt et ressuscite somatique. Les qualifications de cet état somatique auront cependant évolué. Avant la mort, le sôma est dit corruptible, sans valeur et faible. À la résurrection, il se dresse (c'est le sens littéral du verbe ressusciter, ou egeirô employé ici) incorruptible, rayonnant de gloire et de puissance. Il a été transformé par l'action de Dieu, mais était-ce à partir d'un état vil et méprisable?

Le mépris du corps et de la chair en Occident a assez vécu pour avoir influencé nos traductions. Même si on l'imputait à l'influence de Paul luimême, à travers une mauvaise lecture de son emploi du mot chair! Au v. 43a, le syntagme "semé dans l'atimia" devient, selon les traducteurs: "semé méprisable » (TOB), « on sème de l'ignominie » (BJ). Par valorisation de sa gloire à la résurrection, on dévalorise l'état antérieur du corps. Et c'est méprisable, ignominieux que le corps passera dans le discours moral. Or atimia, avec son a privatif devant timia, ou valeur, prix, honneur, respect, n'a pas forcément la valence la plus honteuse du spectre de ses significations propres. C'est le contexte qui indique le choix de la nuance juste. Dans la même épître (1 Co 12,14-26), Paul expose longuement, littéralement "de la tête aux pieds", la merveilleuse harmonie entre les membres d'un même corps. Aucun n'est négligeable; tous concourent au bon fonctionnement de l'ensemble, tous sont indispensables. Néanmoins, aux vv. 22-24, la traduction, au sujet des membres dits 
moins importants et plus faibles qu'on entoure de plus de soins, dérive vers des «membres moins honorables", "indécents", qu'on recouvre de vêtements. Elle nous amène à décoder des "membres moins importants", qui ne sont pas nommés, en "organes sexuels". L'appareil reproducteur et la relation sexuelle ont-ils une valeur marginale dans le corps humain? Si nous lisions "cheveux" au lieu d'organes sexuels? Dans l'antiquité, on leur accordait autant d'honneur et de soins que dans nos salons de coiffure actuels.

À partir d'une conception du corps rectifiée, nous pourrions traduire autrement, sans trahir le texte de départ. Qui, de nous ou de Paul, pratique le mépris du corps? Paul répond, pour sa part, dans le contexte immédiat, de 6,12 à 7,40 , à propos des relations sexuelles, de leurs débordements et de leur exercice à l'intérieur des règles sociales et dans l'optique de la vie chrétienne. Ses considérations sur la porneia (fornication ou débauche) nous ont valu ses plus belles phrases. "Le corps (sôma plutôt que sarx dans toutes ces citations) n'est pas pour la débauche, il est pour le Seigneur et le Seigneur est pour le corps." (1 Co 6,13) "Ne savez-vous pas que vos corps sont les membres du Christ?" (v. 15). "Fuyez la débauche. Tout autre péché commis par l'homme est extérieur à son corps (i.e. à lui-même). Mais le débauché pèche contre son propre corps. Ou bien ne savez-vous pas que votre corps est le temple de l'Esprit Saint...? (vv. 18-19). Glorifiez donc Dieu en votre corps. » (v. 20) Le même respect pour le corps de l'autre régira l'exercice, et non l'interdiction, des relations conjugales.

À la défense des traducteurs, il faut ajouter que nous savons peu de choses des notions de décence, de pudeur et de honte pour Paul et son époque. La Bible contient également des séries dites d'impuretés et d'abominations en matières sexuelles, codifiées au Lévitique surtout. Elles ne s'enracinent pas dans le mépris du corps ou dans un pré-catalogue d'êtres et de pratiques dégoûtants de nature. Elles obéissent à d'autres paramètres de classification, dont le plus évident est la catégorie même/autre et les possibilités prescrites ou interdites de s'unir tantôt au même, tantôt à l'autre. Les verdicts selon le pur et l'impur sont affaire de séparation pour consécration à Yahvé, lui le Séparé, lui le Saint, et non de qualifications morales ${ }^{3}$.

3 Dans Pouvoirs de l'horreur. Paris, Seuil (Points), 1980, au chapitre "Sémiotique de l'abomination ", Julia KRISTEVA a tenté un début d'analyse du pur/impur dans le Lévitique. De la pratique sémiotique, elle passe en théorie analytique et conclut que l'impur fondamental déterminant tous les autres est la fusion avec la mère, dont il faut se séparer pour construire son identité et son autonomie. 
On sait combien Paul s'est distancé de cette division du monde en pur/impur, lui qui avait pourtant vécu de la plus stricte observance de la Loi mosaïque jusqu'à l'incident du chemin de Damas. On le sait par son opposition formelle à Pierre et à Jacques à propos des interdits alimentaires qui constituaient son premier niveau d'application et qu'il refusait d'imposer aux convertis de chez les païens. "J'en ai la conviction dans le Seigneur : rien n'est impur en soi...(Rm 14,14$) \ldots$ car le Règne de Dieu n'est pas affaire de nourriture ou de boisson (v. 17). Ce n'est pas un aliment qui nous rapprochera de Dieu : si nous n'en mangeons pas, nous ne prendrons pas de retard; si nous en mangeons, nous ne serons pas plus avancés. » (1 Co 8,8)

Quant au corps, à sa réalité et ses fonctions, la correspondance paulinienne ne conserve aucune trace de son infériorisation, encore moins d'une attitude méprisante à son égard. Dans le passage que nous lisons, c'est le même sôma qui se voit attribuer incorruptibilité, gloire et puissance, corruptibilité, situation modeste et faiblesse. La suite des versets, en confirmant l'acception de sôma comme expression du tout de la personne humaine, éclairera aussi l'acception du terme sarx responsable de graves malentendus dans le discours chrétien sur les "choses de la chair ».

Les deux listes parallèles d'attributions du sôma avant et après la résurrection sont résumées par le v. 44 : semé sôma psuchikon, il ressuscite sôma pneumatikon. Nous touchons le sommet du texte, la réponse à la question de départ : "Insensé! (v. 36), il existe un sôma pneumatikon comme il existe un sôma psuchikon. " (v. 44b) Elle tient dans la création de deux adjectifs à partir de psuchè et de pneuma : psychique et pneumatique.

"Psychique " n'a rien à voir avec les développements actuels de psychanalyse et psychologie, ni avec l'équivalent lexicographique de psuchè, c'est-à-dire l'âme. C'est le parallélisme établi par la clé de lecture offerte dans la citation de Gn 2,7 qui nous l'apprend:

s'il y a un corps psychique il $y$ a aussi un corps pneumatique

Comme il est écrit :

le premier Adam fut

le dernier Adam

une psuchè

un pneuma

vivante

créateur de vie 
Retrouvons-nous en Adam l'âme à la grecque, une créature animée (du latin : anima pour âme)? Non, l'Adam de la Genèse n'est pas un composé de corps et d'âme mais une néfesh. À son tour, la néfesh hébraïque n'est pas le principe vital qui anime le corps humain, mais la personne humaine corporelle (aussi l'animal) en tant que vivante.

La réponse de Paul s'énonce, au niveau des personnalités corporatives : en Adam, l'être humain est un sôma psychique; dans le Christ, l'être humain devient un sôma pneumatique. De nouveau, la persistance de l'emploi de sôma après la résurrection étonne. Elle pose l'existence d'un corps spirituel (de l'équivalent latin de pneuma: spiritus) ou spiritualisé, d'une matière devenue esprit, ce qui est une contradiction logique en langue et civilisation grecques. Elle pose surtout la notion qu'en anthropologie paulinienne le corps n'est pas une partie du tout humain, incompatible avec sa spiritualisation progressive et "larguable » dans la résurrection, mais le tout d'un être corporel qui, d'abord psychique en Adam devient pneumatique à son passage à l'in-corpo-ration dans le Christ.

La construction de l'opposition psychique/pneumatique a encore plus à nous apprendre. Telle que réfléchie de $\mathrm{Gn} 2,7$, sa formulation ne peut se transcrire en opposition âme/esprit, littéralement correcte au niveau de la langue grecque, corps/esprit ou chair/esprit. Si nous souhaitons la lire à travers l'anthropologie grecque, il faut écrire : corps et âme psychiques par rapport à corps et âme pneumatiques.

Nous apprenons de cette construction que Paul oppose ici deux états possibles de la personne humaine, qu'il désigne par "corps", deux registres de vie. Encore ici, nous pourrions les transcrire en état de " nature " et état dans le Christ, à condition de ne pas oublier que la dichotomie nature/surnature n'existe pas dans la Bible. Ce que nous appelons surnature et spirituel par rapport à matériel n'existent pas sinon dans ce que nous appelons nature et matériel. Psychique/pneumatique comme attributions d'un seul et même corps demeure la seule distinction que Paul établit dans sa vision de l'être humain.

Les versets 46 à 49 développent la citation de la Genese. Ils nous amènent, par la discursivisation du passage entier, à la conclusion que l'image du Christ, lui-même image parfaite de Dieu (2 Co 4,4;3,18) porte sur le tout du moi humain corporel. Nous rejoignons $1 \mathrm{Co} 6,13 \mathrm{~b}$ : «le corps est pour le Seigneur et le Seigneur pour le corps ". Nous nous éloignons de l'univers des Pères de l'Église où la relation à Dieu se jouait à la fine pointe de l'âme, où tout l'effort de la vie humaine consistait à dégager l'image de Dieu enfouie au fond de cette âme envahie par le péché. Heureusement, nous n'avons pas à renoncer aux magnifiques écrits de ce type dans la littérature patristique, mais à voir sur quelle dichotomie 
corps/âme ils sont étayés. C'est contre leurs déviations, du côté de l'indignité et des abjectes servitudes du corps à réprimer, que le réalisme biblique apporte un bienheureux assainissement.

Le maniement littéraire des lexèmes sôma, sarx et psuchè, des adjectifs dérivés, des oppositions qui les articulent ont éclairé la charge sémantique des lexèmes eux-mêmes. Elle ne relève pas du dictionnaire mais de l'action de l'énonciateur; création originale en ce cas. Surtout, la mise en discours de la question de départ, jugée «insensée » en celui qui la pose, Corinthien fictif, ou de papier, ou résident de la Corinthe connue de Paul, permet de constater que sôma désigne le tout de l'être humain.

Sarx a la même extension, c'est-à-dire l'être humain entier en tant qu'inscrit dans la finitude, et non cette part délimitée en lui appelée: sexualité, sensualité, avidité pulsionnelle. Cette conception se vérifie dans des textes de lecture difficile, qui en sont, à leur tour, éclairés. Ainsi Paul peut-il parler ailleurs de "sagesse charnelle " $(2 \mathrm{Co} 1,12)$, d'intelligence charnelle $(\mathrm{Col} 2,18)$, contradictions dans les termes, et qualifier d'ouvres de la chair des dérogations comme l'idolâtrie et les dissensions et les factions hors de tout lien avec des passions sexuelles coupables.

Si la chair s'oppose à l'esprit en $G a 5,17$, c'est à la façon du psychique et du pneumatique, à la façon de deux registres différents. Paul reprochera souvent à ses chrétiens, déjà passés au second, de vivre encore "selon la chair ", tout en admettant que, tant que nous sommes dans la chair, c'està-dire en-deçà de la résurrection, nous sommes soumis à ses limites, surtout à la défaite devant péché, mal et mort, tout libérés par le Christ que nous soyons.

Si devant tel sôma et telle sarx, on pose à nouveau la question de la résurrection, la réponse paulinienne devient la résurrection du sôma, du tout de l'être humain, qui ne saurait être autre que somatique à la différence de Dieu et des esprits bons ou maléfiques. Elle sera "pneumatisation" de toute la personne. Par définition, cependant, elle ne saurait être dite résurrection de la chair, la résurrection complète, commencée au baptême chez Paul, consacrant la fin de la finitude. Aussi le corpus paulinien n'utilisera-t-il jamais que résurrection de ceux qui sont morts et résurrection d'entre les morts ${ }^{4}$.

4 Dans l'Église catholique romaine, un document de la Congrégation pour la doctrine de la foi, daté de mars 1984 et reproduit dans la Documentation catholique, septembre 1984 , no $1880,849-850$ prône le maintien de la traduction littérale du "Carnis resurrectionem » du Symbole des Apôtres en " résurrection de la chair ». Il la déclare " plus explicite dans l'affirmation de cet aspect particulier de la résurrection ". Pour des populations de chrétiens qui fonctionnent selon l'anthropologie grecque, elle 
Dans la perspective paulinienne, l'interrogation de certaines sciences actuelles, soit : "Comment la subjectivité est-elle liée à un corps, à un objet physique, matériel?", ne se pose même pas. Cette formulation suppose une anthropologie dualiste où le matériel s'oppose au spirituel. De même dans la conception fréquente du corps comme expression du soi, comme dimension de la relation à soi, aux autres et au monde affleure l'opposition entre extérieur et intérieur. Ce deuxième cas correspond encore moins bien à la réalité biblique où Dieu tout-relation est présenté comme n'ayant pas de corps.

En bref, dans l'anthropologie unitaire de Paul, le terme corps désigne la personne humaine tout entière, le sujet humain, et non sa composante physique, matérielle, vivant en symbiose avec une composante spirituelle, ou hôte d'une âme selon les écoles philosophiques ${ }^{5}$. Ce sujet peut vivre et grandir sur deux registres différents, le psychique et le pneumatique, le second s'ouvrant avec l'identification au Christ. Dans cette optique, encore une fois, il n'existe pas d'autre du corps.

\subsection{Les évangiles}

Comme chez Paul, en 1 Th 5,23, une énumération de termes peut nous induire en erreur sur l'anthropologie des évangiles. À la question d'un scribe : "Quel est le plus grand commandement de la Loi, le premier? », le Jésus de Matthieu répond en 22,37: «Tu aimeras le Seigneur ton Dieu de tout ton cour (kardia), de toute ton âme ( $p s u c h e ̀)$, de toute ta pensée "(dianoia, raison, esprit). Le Jésus de Marc ajoute : "de toute ta force » (ischus) (Mc 12,30), celui de Luc intervertit pensée et force dans

renvoie, en effet, au corps, mais au niveau populaire, la théorie de l'âme subsistante venue de Thomas d'Aquin relance exactement la même question que 1 Co 15,35: Avec quel corps, les morts reviennent-ils?

5 Quelques passages des épitres apportent une dissonance dans ce tableau. Nous n'avons pas à les étudier ici, mais à signaler leur existence et l'intérêt de les revoir à la lumière de la vision unitaire. Les oppositions homme intérieur/homme extérieur (2 Co 4,16) et vieil homme/homme nouveau (Ep 4,22-24; Col 3,9-10) n'y font pas vraiment exception. 2 Co 5,1-10 nous ramène plus crûment au dualisme corps/âme : «... tant que nous habitons dans ce corps, nous sommes hors de notre demeure, loin du Seigneur $(v, 6) \ldots$ nous préférons quitter la demeure de ce corps pour aller demeurer auprès du Seigneur " (v. 8). Emprunt de Paul à l'anthropologie de ses correspondants, avec visée pédagogique? Nous n'en savons rien. L'étude de l'entrelacs des images de la demeure à quitter ou à investir et du vêtement à laisser tomber ou à revêtir nous réserverait peut-être des surprises dans la ligne de la note fine de la TOB en 5,1: " on emménage dans un habit, et on s'habille d'une maison ". 
l'ordre d'énumération ( $L c 10,27)$. La source de cette phrase au $D t 6,5$ porte, en hébreu : cœur (lèv), être ou vie (néfesh) et une variation du mot force qui pourrait se traduire aussi par ferveur, intensité; dans le grec de la Septante reviennent: pensée, âme et force (dunamis), mais sans le cœur, omission fidèle à l'anthropologie hébraïque où l'on pense avec son cœur.

La réponse de Jésus signifie: "Tu aimeras ton Dieu de tout toimême! " Nous aurions là, avec ses variantes, la vision de la personne humaine selon les évangiles, une vision toute spiritualisée, puisque corps et chair en sont absents? Une vision spiritualisée, spirituelle sans l'Esprit, car pneuma est également absent?

Les trois grands oubliés sont pourtant bien présents ailleurs dans les textes, mais sans la cohésion et la cohérence de leurs acceptions auxquelles nous a habitués le corpus paulinien. La chair désigne tantôt toute la personne (Mt 24,22; In 17,2), tantôt est opposée à l'esprit dans des passages hybrides qui peuvent se lire et en anthropologie unitaire et en anthropologie duelle ou dualiste (Mt 26,41; Lc 24,39; Jn 6,63). De même pour le corps dont l'opposition à l'âme en $M t 10,28$ ne peut être ramenée à la vision unitaire: "Ne craignez pas ceux qui tuent le corps, mais ne peuvent tuer l'âme; craignez plutôt celui qui peut faire périr âme et corps dans la géhenne". L'évangile de Luc, pourtant le plus grec des deux évangiles, va corriger la phrase en supprimant âme et la resituer en anthropologie hébraique : "... ne craignez pas ceux qui tuent le corps et qui, après cela, ne peuvent rien faire de plus... craignez celui qui, après avoir tué, a le pouvoir de jeter dans la géhenne» (Lc 12,4-5).

Les occurrences de psuchè ont cependant gagné en clarté depuis qu'on ne les traduit plus par âme mais par vie : "Que servira donc à l'homme de gagner le monde entier, s'il perd sa vie " (Mt 16,25-26). Les emplois de pneuma restent les plus ambigus. Ils vont de: «Heureux les pauvres en esprit!" (Mt 5,3), à la catégorie d'êtres appelés esprits impurs, à l'Espritsaint, en passant par les expressions "gémir en son esprit " $\left(\mathrm{Mc}_{\mathrm{c}}, 12\right)$ et «tressaillir en son esprit» (Lc 1, 47).

Sous réserve d'étude plus poussée, il semble que deux conceptions de la personne s'entremêlent, dans l'ambivalence de leurs frontières respectives. À la différence de la correspondance de Paul, le corpus des évangiles nous amène en sol moins unifié. Mosaïque de traditions locales, temporelles, orales et écrites, d'auteurs collectifs et individuels, de transmissions variées, il véhicule des traces de plusieurs cultures. Relu par Paul, deviendrait-il plus cohérent? Or, nous savons que l'acception du même vocabulaire varie d'un écrit à l'autre dans le Nouveau Testament. 
Nous savons également que les épîtres ne découlent pas des évangiles comme le commentaire du commenté. Les deux corpus mettent en discours la même tradition chrétienne des origines, à laquelle ils participent d'ailleurs, l'un sous mode narratif, l'autre sous mode figuratif, d'un figuratif de plusieurs niveaux, l'un et l'autre déjà "théologisés". Nous poserons donc plutôt à la forme du récit des évangiles la question de la condition corporelle de Jésus dans l'optique de celle de la subjectivité. En lecture de surface, que nous disent les évangiles de son expérience corporelle aux moments de l'incarnation, de la transfiguration, de la Cène, de la mort et de la résurrection? Nous autorisent-ils à voir dans le personnage Jésus un autre de son corps?

\subsection{1 À l'incarnation}

Selon Matthieu, Jésus fut engendré de Marie $(1,16)$. En songe, l'Ange du Seigneur apprend à Joseph, son époux, que : «ce qui a été engendré en elle provient de l'Esprit saint » $(1,18.20)$. En Luc, l'ange Gabriel tient le même langage à Marie : "L'Esprit saint viendra sur toi et la puissance du Très-Haut te couvrira de son ombre; c'est pourquoi ce qui naîtra sera saint, appelé fils de Dieu " $(1,35)$. Plus loin, le récit déclare : "l'enfant grandissait et se fortifiait, plein de sagesse et la faveur de Dieu était sur lui» $(2,40)$; il avait eu la même phrase à propos de Jean Baptiste, dont la naissance est liée à celle de Jésus, mais avec une variante: «l'enfant grandissait et se fortifiait en esprit $»(1,80)$.

Conçu, engendré par Zacharie et Elisabeth, Jean fut rempli de l'Esprit saint dès le sein de sa mère $(1,15 b)$ et il devait marcher sous le regard de Dieu, "dans l'esprit et la puissance d'Élie " $(1,17)$. Conçu directement par la puissance de Dieu, par l'Esprit saint, Jésus a un rapport supérieur à l'Esprit, qui dépasse l'esprit prophétique. Cependant, il n'est pas dit incarnation de l'Esprit ou, en aucune manière, produit d'un corps humain animé par l'Esprit.

En Jean, tout au contraire, un Verbe, un logos, qui était auprès de Dieu, qui était Dieu, en qui était la vie, vint vers l'humanité $(1,1-4)$. Et le Verbe fut chair, le logos est devenu sarx, et nous l'avons vu, lui, le monogène du Père $(1,14.18)$. Ici le Logos divin s'est in-carné lui-même, mais à la façon des évangiles de Matthieu et de Luc, il n'est pas dit non plus produit de l'union d'une chair avec le Verbe qui lui tiendrait lieu d'âme, de principe vital.

\subsection{2. À la transfiguration}

Une longue tradition, sans doute dérivée des versions latines de la Bible, nomme "transfiguration "l'épisode raconté en $M t$ 17,1-8; Mc 9,2-8; 
Lc 9,28-36. Le grec de Matthieu et de Marc porte: Jésus fut métamorphosé devant eux (métamorphôtè). Luc ne nomme pas le phénomène mais le décrit. Sa description rejoint celles des deux autres Synoptiques. Les vêtements de Jésus deviennent resplendissants, d'un blanc aussi éclatant qu'une lumière fulgurante, son visage comme le soleil $(\mathrm{M} t)$, d'un aspect autre. Le résultat de la métamorphose est si beau que les spectateurs Pierre, Jacques et Jean, sont, pour leur part, dans l'euphorie malgré leur effroi.

La relecture de l'événement par la seconde épître de Pierre $(1,16-18)$ le qualifie de perception oculaire de la "majesté " du Seigneur Jésus Christ, car il reçut de Dieu le Père honneur et gloire quand une voix portée par la gloire magnifique vint à lui en ces termes : "Celui-ci est mon fils, mon bien-aimé, en qui j'ai trouvé mon plaisir". Dans les Synoptiques, après la phase d'anamorphose ${ }^{6}$, avec l'arrivée de la nuée qui couvre de son ombre toute cette splendeur, Matthieu ajoute que Jésus touche les trois disciples tombés face contre terre et qu'ils redescendent de la montagne ( $\mathrm{Mt}, \mathrm{Mc})$.

Malgré son caractère saisissant, les textes décrivent cette expérience comme corporelle, les vêtements mêmes y participent, et non comme affleurement de l'âme ou prise de possession de Jésus par l'Esprit ou fusion avec Dieu le Père, ou passage à une autre forme, fût-elle supérieure. Les trois spectateurs la vivent également corporellement, de leurs yeux, de leurs oreilles, de leur agitation. Un corps, c'est-à-dire une personne humaine, a soudainement et momentanément été vu autrement, dans toute l'extension de l'honneur reçu de Dieu, à l'intérieur d'une relation filiale privilégiée. La transfiguration de Jésus est le moment d'épiphanie de l'événement de l'incarnation?

\subsection{3 À la Cène}

Dans la recherche du corps à la Cène, il faut commencer par oublier ce qui deviendra dans les siècles postérieurs paroles de l'institution de l'eucharistie, réduction des symboles à la messe et interprétations confessionnelles. Les récits restitués à eux-mêmes, quelle est la situation du corps de Jésus à l'intérieur de leur cadre? Les lectures oscillent entre anthropophagie, symbolisme et geste prophétique qui, dans la Bible,

Voir l'intéressante analyse sémiotique de la transfiguration dans François Martin, Pour une théologie de la lettre. L'inspiration dans les Écritures. Paris, Cerf (Cogitatio fidei, 196), 1996, pp. 151-162 et 249-257 sur le transfert du " corps dans l'ordre du langage en acte d'énonciation, l'ordre même de la parole ".

7 Formulation de François MARTIN, ibidem, p. 391, note 1. 
accomplit ce qu'il représente. Nous nous en tiendrons aux dispositifs de la mise en discours.

Le retour aux textes nous amène à sa version écrite la plus ancienne, que nous traiterons cependant en relation synchronique avec celles des évangélistes, parallèle parmi des parallèles. En Paul, dans la première épître aux Corinthiens $(11,24-25)$, sur le pain qu'il a rompu, Jésus dit : "Ceci est mon corps, qui est pour vous" (littéralement : le pour-vous); sur la coupe : "Cette coupe est la nouvelle alliance en mon sang». Dans les Synoptiques, sur le pain qu'il a donné à ses disciples Jésus dit : "Ceci est mon corps, qui est donné pour vous" (Lc 22,19); "Prenez, ceci est mon corps » (Mc 14,22); «Prenez et mangez, ceci est mon corps» (Mt 26,26). Sur la coupe: "Cette coupe est la nouvelle alliance en mon sang, qui est répandu pour vous " $(L c 22,20)$; "Ceci est mon sang de l'alliance, qui est répandu pour beaucoup » (Mc 14,23); "Buvez-en tous, car ceci est mon sang, celui de l'alliance, qui est répandu pour beaucoup en vue de la rémission des péchés. "

Les formules varient, s'éloignant ou se rapprochant du support métaphorique d'un repas bien concret. Jésus sera arrêté quelques heures plus tard et mourra le lendemain. Le vin partagé, ou le sang répandu, figure cette mort, avec le sens décuplé en "alliance pour une multitude " qu'il lui confère. Identifié à lui, le pain distribué aux convives représente le don de lui-même, son amour pour eux ultimement. Le syntagme "pour vous " revient constamment. "Je suis pour vous et c'est pour vous que je me donne dans cette mort que j'accepte. "Dans ces textes, corps ne peut se lire en anthropologie dualiste, sous peine que Jésus offre son corps, sa composante physique, et non son âme, son esprit, l'essence de lui-même. Corps coïncide avec " je " et désigne Jésus. Enfin, malgré le parallélisme des paroles sur le pain et le vin, l'articulation corps/sang n'éclaire pas la lecture. Même si on place la figure de la mort dans leur séparation, le corps vidé de son sang reste Jésus lui-même, et le sang le support du rite d'alliance dans la littérature biblique.

L'évangile de Jean raconte le repas d'adieu de Jésus et de ses disciples, mais ne rapporte pas ses gestes et ses paroles sur le pain et le vin. L'essentiel d'une communion à lui sous forme de nourriture apparaît cependant en 6,22-59. Après une multiplication des pains qui lui a attiré des fidèles trop intéressés par la commodité de ses pouvoirs, Jésus veut les hausser au niveau d'une alimentation supérieure. "Le pain que je donnerai, c'est ma chair donnée pour que le monde ait la vie.... si vous ne mangez pas la chair du Fils de l'homme et si vous ne buvez pas son sang, vous n'aurez pas en vous la vie... car ma chair est vraie nourriture et mon sang vraie boisson" $(6,51-55)$. Jésus ne décode pas ces paroles pour les auditeurs scandalisés qui le quittent brusquement. Pour ceux qui restent, mais 
avec inquiétude, il ajoute: "C'est l'esprit qui vivifie, la chair ne sert de rien. Les paroles que je vous ai dites sont esprit et vie" (v.63). Comprenez sous le mode charnel, sans esprit dans tous les sens du mot, et vous reculerez devant l'anthropophagie entendue par vos oreilles.

La sarx a remplacé le sôma des récits de la Cène. L'acception totalisante du terme reste la même, et c'est à l'identification à lui que Jésus invite dans le reste de la séquence, au moyen de la comparaison avec la nourriture. Le jeu sur le sang est différent. Ce n'est plus le sang de l'alliance, mais le sang de l'expression chair-et-sang désignant ailleurs toute la personne sous son aspect de finitude et de fragilité.

\subsection{4 À la mort}

Après la mise en scène prophétique, hiératique de la mort, sa mise en action effective est éminemment psychosomatique. Après le sublime de la Cène, l'expérience corporelle de l'angoisse caractérisée devant la mort fond sur Jésus à Gethsémani, jusqu'à la sueur de sang en $L c 22,44$, jusqu'à l'écrasement par terre et aux chutes répétées, jusqu'à l'aveu en $\mathrm{Mc}$ et $\mathrm{Mt}$ que nos traductions rendent par : "Mon âme (psuchè) est triste jusqu'à la mort ». Comme il s'agit du verset 6 du Psaume 42 rapproché du verset 5 du Psaume 41 dans la Septante, il faudrait lire: néfesh sous psuchè, ce qui revient à dire: "Je suis triste à en mourir ». Devant les trois mêmes témoins, Pierre, Jacques et Jean, Jésus subit une transformation à l'inverse de la transfiguration en majesté, mais qui est aussi épiphanie de l'incarnation.

Quant au conseil aux trois disciples: "Veillez et priez pour ne pas entrer en tentation : l'esprit est ardent mais la chair est faible", il se lit parfaitement en anthropologie hébraïque, si à la façon de Paul, on oppose un registre charnel à un registre pneumatique et non, à la grecque, les deux composantes de chair et d'esprit.

La machine pénale appliquera ensuite tous les sévices corporels qui causeront la mort du condamné. En croix, Jésus répond au "bon larron ": "En vérité, je te le dis, aujourd'hui tu seras avec moi (et non avec mon esprit ou avec mon âme immortelle) en paradis " (Lc 23,43). Le mot paradis étonne : ni shéol à l'hébraiqque, ni Hadès à la grecque, mais paradis à la persane. Anthropologie, cosmologie, cartographie de l'au-delà sont liées et un emprunt de ce genre témoigne d'une perméabilité aux influences étrangères dont il faudrait tenir compte dans une étude plus poussée de la question du corps dans les évangiles.

En Lc 23,46, à la neuvième heure, Jésus crie fortement : "Père, dans tes mains je remets mon esprit $\%$. De nouveau un verset de psaume (Ps 31,6; Septante 30,5) où pneuma recouvre donc l'hébreu rouah. Quel 
esprit Jésus remet-il en mourant? L'Esprit saint, son esprit ou son âme, son souffle d'être vivant? La suite de Luc et les parallèles parlent de souffle : « ayant dit cela, il expira "; " Jésus ayant jeté un grand cri expira » $(M c)$; « ayant clamé à grand cri, il laissa partir son esprit (son souffle?) » $(M t)$; «penchant la tête, il rendit l'esprit » (Jn). Sauf en Lc et Mc qui emploient «expirer", les verbes choisis par $\mathrm{Mt}$ et Jn, réciproquement délivrer (aphièmi) et livre (paradidômi) l'esprit, ouvrent cependant la porte à tous les commentaires qui souhaiteraient trouver ici le moment du don de l'Esprit à l'humanité et vérifier cette hypothèse.

Les séquences suivantes, jusqu'à l'aurore du premier jour de la semaine, multiplient les éléments textuels qui servent à vérifier et à asserter la mort de Jésus, comme le coup de lance du centurion en Jean, la permission d'ensevelir accordée par Pilate, la descente de croix, la toilette du corps devenu cadavre, la mise au tombeau, les scellés sur la pierre d'entrée et les soldats qui montent la garde en Matthieu. Enfin, tout ce qui concerne cette mort se lit, sans difficulté, en anthropologie unitaire hébraïque.

\subsection{5 À la résurrection}

Si la transfiguration a été racontée, la résurrection ne sera connue que par ses effets. À l'arrivée des femmes, tôt le lendemain du sabbat, dont l'observance les avait retenues dans Jérusalem, un tombeau ouvert, des bandelettes, un suaire, mais de corps de Jésus, point. Un ou deux anges, selon les versions, leur annoncent qu'il a été "éveillé des morts. Des verbes également au passif diront bientôt après qu'il a été manifesté, montré, vu par plusieurs. "Mais avec quel corps?", interrogeraient les Corinthiens à la suite de leurs penseurs.

Selon la finale ajoutée à Marc, Jésus " a été révélé sous une autre forme (morphè)". Effectivement, on ne le reconnaît pas au premier regard. Marie de Magdala le voit debout dans le jardin près du tombeau et le prend pour le jardinier. Selon $L c$ 24,16.31, les yeux des disciples d'Emmaüs " étaient empêchés de le reconnaître " et s'ouvrirent à sa façon de rompre le pain à table, mais, lui, à ce moment devint invisible de devant eux. Il apparaît au milieu de ses diciples, dans une pièce portes closes, disparaît avec autant de rapidité. Pour qu'on le reconnaisse, il montre la marque des clous dans ses mains, sur ses pieds et la trace de la lance à son côté, vérifiables au doigt et à la main. Il demande qu'on le touche car " un esprit (pneuma) n'a ni chair (sarx) ni os, comme vous voyez que j'en ai... Avez-vous ici quelque aliment? Eux lui offrirent un morceau de poisson grillé et, l'ayant pris, il le mangea devant eux " ( $L c$ 24,39-43). C'est lui qui prépare le déjeûner sur la grève du lac de Tibériade, pour des 
convives dont "aucun n'osait lui demander : "Qui es-tu? " sachant que c'était le Seigneur " (Jn 21,12).

Ce mélange de connaissance et d'in-connaissance a quelque chose de déconcertant. Cet être présent hors des contraintes spatiales des corps humains, parle d'un passé où il était encore avec eux $(L c 24,44)$, du moment où il partira tout en restant toujours avec eux, d'un retour certain malgré une présence constante. Décider de la non-historicité de récits que les générations antérieures de chrétiens ont lus littéralement, au nom d'une autre conception de l'histoire et de la normativité de la Bible, laisse ouverte la question importante : historiques ou mythiques, que nous disentils? Quant à l'expérience corporelle du corps des apparitions, ils mettent l'accent, contre toute attente, sur sa "physicalité ». On oppose parfois en exégèse la spiritualisation ou pneumatisation du corps à la résurrection, chez Paul, à sa matérialisation dans les évangiles, surtout chez Luc. Mais que savons-nous d'un corps entièrement transformé par l'esprit? Ce que nous avons en mains, c'est que le Nouveau Testament l'appelle toujours corps et que ce mot exprime la personne humaine dans tous les états de son aventure existentielle.

À travers les récits des évangiles, nous sommes partis à la recherche d'indices sur la valeur de la condition corporelle du personnage Jésus, aux moments clés qui la mettent en jeu et en discours. Nous pouvons certes conclure qu'il est impossible de décrire la trajectoire de sa vie comme une trans-corporalité, au sens de passage par un corps, que nous en fixions le point de départ dans le Verbe auprès de Dieu, comme en Jean, ou comme le fruit d'une intervention de l'Esprit sur terre, comme dans les Synoptiques. Malgré l'attrait conceptuel et esthétique de la formalisation descente/remontée, le texte ne nous présente pas un Logos - Parole subsistante entrant dans un corps de chair ("Quel corps? ", diraient encore les Corinthiens dont nous sommes) en ressortant par la mort, ressuscitant Parole et laissant dans le monde, à sa réintégration au sein du Père, des "serviteurs de la Parole» (Ac 6,4). Dans un sens, et plusieurs sens, il est juste de dire que le corps du Christ est devenu Parole. Cette affirmation ne rend pas pleinement compte à mon avis, de l'expérience corporelle décrite. Le Christ de ces évangiles n'a pas emprunté un corps, puis ne s'en est pas délesté dans la mort pour poursuivre sur un mode spirituel. Dans la Bible, la mort n'est pas que mort du corps, mais de toute la personne, et la résurrection, de la même façon, resurrection de toute la personne, en tant que corps maintenant complètement "pneumatisé ".

Ces considérations relancent aussi la question plus large de la valeur à accorder à l'expérience corporelle manifestée dans les textes, expérience portant la marque d'une époque révolue, issue d'une aire culturelle circonscrite. De même pour le passage du corps à la Parole, s'ouvre une 
interrogation sur l'acception de "Parole " adoptée en exégèse, en théologie, en théorie sémiotique, en psychanalyse, et à l'intérieur de ces disciplines selon les différentes écoles.

Pour le moment, nous concluons, de façon minimale, qu'en lecture de surface, dans les évangiles comme dans la correspondance de Paul, nous ne trouvons pas d'autres du corps. Malgré certaines exceptions, peu nombreuses, ces écrits se lisent au mieux en anthropologie hébraique unitaire. Le corps y nomme le sujet humain en tant que tel dans l'échelle des êtres, ni Dieu, ni esprit, ni ange, ni démon.

\section{L'autre du corps : l'intersubjectivité et l'être chrétien}

Le sujet humain existe, sa subjectivité est celle de son corps, est son corps ou n'existe pas. Elle n'est pas liée à un objet qui, s'il est dit matériel, suppose qu'elle ne l'est pas. La distinction matériel/spirituel ne s'applique pas dans cet univers en relation directe, complète avec Dieu où ce que nous appelons matériel participe du spirituel, où le spirituel se vit dans le matériel, sous peine de ne pas être lui non plus.

S'il y a un autre du corps, c'est un autre corps, dans une relation d'inter-subjectivité plus étroite que celle de nos sociétés. La primauté du groupe, du clan, du peuple relativise la valeur de l'individu; il vit immergé dans des personnalités corporatives, par exemple, en Adam, en Moïse, dans le second et l'ultime Adam. Par contre, cette intégration lui laisse conscience de soi et responsabilité personnelle devant Dieu, la loi, ses frères et sœurs en humanité. Cette dernière relation, c'est-à-dire le rapport au prochain, est soigneusement codifée et relève d'une haute moralité et dans l'Ancien Testament et dans le Nouveau, même si le système patriarcal maintient en place des oppressions que ne rejoignent pas les déclarations de principes.

L'être-chrétien également se vit en intersubjectivité, et non en juxtaposition de disciples de Jésus engagés dans un développement spirituel individuel. Selon Paul, devenir chrétien, c'est être identifé au Christ par le baptême dans sa mort-résurrection et, en lui, entrer en identification avec les autres chrétiens. C'est être in-corpo-ré à l'Église qu'il décrit précisément à l'aide de la métaphore du corps humain. "En effet, le corps est un et pourtant il a plusieurs membres; mais tous les membres du corps, malgré leur nombre, ne forment qu'un seul corps : de même le Christ. Car nous avons été baptisés dans un seul Esprit pour être un seul corps.... (1 Co 12,12-13). Suit un dialogue alerte entre pied, main, oreille, et cil : "L'œil ne peut dire à la main : "Je n'ai pas besoin de toi ", ni la tête dire aux pieds : " Je n'ai pas besoin de vous "... Or, vous êtes le corps du Christ et vous êtes ses membres, chacun pour sa part " (v. 27). 
Paul va donc jusqu'à nommer l'ensemble des chrétiens le Corps du Christ, parfois le Corps de façon absolue (1 Co 11,29), parfois, comme cihaut, le Christ. Vice-versa, le Christ n'est jamais dit l'âme de ce corps qui serait composé de l'assemblée des chrétiens; l'addition de nouveaux membres, leur croissance, c'est-à-dire leur christianisation et "pneumatisation» progressives, l'exercice des ministères communautaires "bâtissent le corps du Christ " (Ep 4,12), amènent le Christ total à la taille de sa plénitude (v. 13). Dieu «l'a donné, au sommet de tout, pour tête à l'Église qui est son corps, la plénitude de Celui que Dieu remplit luimême totalement $»(E p 1,23)$.

À ces sommets, mythique et mystique, Paul porte lui-même son ecclésiologie. La clé de cette magnifique "logie " n'en demeure pas moins le point de comparaison du corps humain. Dans la conception paulinienne, la Tête ne peut dire aux pieds: " Je n'ai pas besoin de vous», et l'êtrechrétien se traduit par l'expression : en un Corps. Ici encore ce Corps-là n'a pas d'autre interne à soi.

\section{Nos corpus exégétiques et théologiques}

À l'aide d'indices de surface dans les textes bibliques et de connaissances déjà établies en exégèse, nous avons pu conclure que, dans la Bible, la personne humaine n'abritait pas un autre de son corps. Selon la pensée sémitique, même exprimée à travers les distinctions anthropologiques de la langue grecque, il n'y avait d'autre du corps qu'un autre corps. Ces constatations nous placent aussi devant un corps autre que celui que nous avons reçu, et vécu! dans la tradition aristotélico-thomiste. Beaucoup de nos discours exégétiques, théologiques, chrétiens en général, construits sur la source biblique ne lui correspondent pas. Beaucoup de culpabilités paralysantes sont nées des malentendus tragiques sur les acceptions de corps et de chair dans un texte reconnu comme normatif pour la conduite de la vie.

Nos lectures ont besoin de toutes les précisions fournies par l'histoire, la philologie, la connaissance des anthropologies contemporaines des dates de rédaction des écrits bibliques. Est-ce à dire que l'anthropologie proprement biblique ainsi dégagée constitue la voie d'évasion des catégories binaires absolues, dualistes et hiérarchisées qui nous enferment, à partir des systèmes conceptuels sophistiqués jusqu'à la vie courante toute banale?

Cette vision de l'être humain est certes rafraîchissante avec sa réhabilitation du corps et de la chair. Et il ne faudrait pas imputer trop naïvement à la pensée grecque un mépris du corps que resitue par ailleurs la beauté apollonienne de la statuaire hellénique. Cette vision différente a 
surtout le premier mérite de nous révéler l'existence de notre propre anthropologie et d'en relativiser les évidences non critiquées. Séduisante, elle n'en a pas moins aussi ses limites. Sur l'au-delà et l'après-mort, par exemple, elle ne fournit aucun instrument de pensée et elle s'est butée elle-même au problème de la rétribution. Ce que nous en avons vu brièvement ici ne permet pas de l'évaluer non plus par rapport au mystère du mal et du péché. En rappelant les acceptions de corps et de chair, nous en sommes restés à ce que Leibniz appelait «l'Adam vague ${ }^{8}$ ».

À condition de ne pas les projeter sur et entre les lignes de la Bible, on peut sereinement continuer à se penser en corps/âme, et penser les réalités bibliques elles-mêmes. À condition, cette fois, de prendre en compte les modalités littéraires des textes, à travers lesquelles se construit le sujet humain instauré dans son statut d'humanité par Dieu, cet autre sujet qui vient à lui. Dans l'acte de lecture, le sujet lecteur amène à son siècle cette intersubjectivité dont il participe. Alors peut varier le vestiaire linguistique dont il revêt corps et chair.

8 Ilya PRIGOGINE et Isabelle STENGERS citent cette appellation prégnante dans La querelle du déterminisme. Philosophie de la science d'aujourd'hui. Paris, Gallimard, 1995, p. 250 : « sous le regard de Dieu, l'Adam qui exista comme premier homme ne pouvait que pécher : le péché originel était inscrit dans sa définition individuelle. Pourtant, souligne Leibniz, nul, y compris Adam, ne pouvait prévoir le péché : quelle que soit la somme des informations que nous ayons pu accumuler à propos d'Adam avant son acte, nous arriverions toujours à la définition d'un "Adam vague", compatible avec la définition d'une infinité d'Adam susceptibles de destins divergents, susceptibles de pécher ou de résister à la tentation, et non pas à la définition individuelle infiniment précise, parfaite, d'où découle la nécessité du péché ". 